\title{
The Effectiveness of Occupational Therapy-Based Sleep Interventions on Quality of Life and Fatigue in Patients with Multiple Sclerosis: A Pilot Randomized Clinical Trial Study
}

This article was published in the following Dove Press journal:

Neuropsychiatric Disease and Treatment

\author{
Malahat Akbarfahimi (iD) \\ Seyed Massood Nabavi (iD) ${ }^{2}$ \\ Benyamin Kor (iD ${ }^{3}$ \\ Leeba Rezaie (iD) ${ }^{4}$ \\ Ethan Paschall (iD ${ }^{5}$ \\ 'Department of Occupational Therapy, \\ School of Rehabilitation Sciences, \\ Neuroscience Research Center, Iran \\ University of Medical Sciences, Tehran, Iran; \\ ${ }^{2}$ Regenerative Biomedicine Department, \\ Cell Research Center, Royan Institute for \\ Stem Cell Biology and Technology, ACCR, \\ Tehran, Iran; ${ }^{3}$ Department of Occupational \\ Therapy, School of Rehabilitation Sciences, \\ Iran University of Medical Sciences, Tehran, \\ Iran; ${ }^{4}$ Sleep Disorders Research Center, \\ Kermanshah University of Medical Sciences, \\ Kermanshah, Iran; ${ }^{5}$ Clinical Psychology, \\ Eastern Michigan University, Ypsilanti, \\ Michigan, USA
}

Purpose: Sleep difficulties are common in patients with multiple sclerosis (MS), which may increase feelings of fatigue, negatively interfere with daily activities, and consequently reduce their quality of life. Studies examining the effects of sleep-targeted interventions in MS are currently limited in the literature. Therefore, we aim to assess the effects of occupational therapy interventions on sleep quality, fatigue, and quality of life in patients with MS

Patients and Methods: In a single-blind, randomized, controlled trial, which occurred between April 2018 and March 2019 in Tehran, Iran, 20 eligible patients with MS were assessed using the Pittsburgh Sleep Quality Index (PSQI), Fatigue Impact Scale (FIS), Fatigue Severity Scale (FSS), and Short-Form Health Survey (SF-36). Patients were allocated randomly into the two following groups: patients receiving care-as-usual for MS $(\mathrm{CAU})$ and patients receiving care-as-usual plus intervention (CAU + intervention). Both intervention groups underwent 2-3 sessions per week lasting 30-45 minutes for 8 weeks and received follow-up assessments. Data were analyzed using independent sample t-tests and Mann-Whitney U tests using SPSS (16 ver.) statistical software.

Results: In the intervention group, sleep quality improved significantly across all items $(p<0.001$, effect size $=0.60)$ except for sleep efficiency and the use of sleep medications. FSS and FIS in the sleep intervention group were significantly reduced $(p<0.001$, effect size $=0.76$ and $p<0.001$, effect size $=0.82$, respectively). The quality of life in the intervention group improved significantly $(p<0.004$, effect size $=0.51-0.76)$ with the exception of the social functioning subgroup.

Conclusion: Although this is the result of a pilot study and more patients should be added, this intervention program demonstrates improvement in sleep quality and quality of life while decreasing fatigue in patients with MS. Adjunction of this program, if results are similar with more patients, to routine occupational therapy (OT) interventions can help improve the rehabilitation program of MS patients.

Keywords: sleep quality, quality of life, fatigue, multiple sclerosis, sleep interventions, occupational therapy

\section{Introduction}

Multiple sclerosis (MS) is a chronic autoimmune, inflammatory, and neurodegenerative disease characterized by axon demyelination in different parts of the brain and/or spinal cord which may cause physical and cognitive symptoms, sensory

Tel $+98|883644| 4$

Fax +988338265255

Email rezaie.phd.ot@gmail.com 
impairments, and depressed mood. ${ }^{1}$ Sleep disorders of multifactorial nature and unknown pathophysiology affect approximately $60 \%$ of patients with $\mathrm{MS}^{2}{ }^{2}$ Several types of sleep disorders are prominent in patients diagnosed with MS such as insomnia, restless leg syndrome (RLS), periodic limb movement disorder (PLMD), obstructive sleep apnea due to respiratory problems, and daily excessive sleeping or narcolepsy. ${ }^{3}$ Sleep disturbance in MS, specifically insomnia, causes excessive limitations in patients' physical and cognitive skills and contributes to increased feelings of fatigue. ${ }^{3,5}$ Additionally, sleep disturbance, when comorbid with depression and anxiety, often leads to diminished participation in social situations and increased dependence on others to accomplish activities of daily living (ADL), work, and leisure activities which can eventually lead to a severe decline in the individual's quality of life (QOL). 5,6 Therefore, early interventions are a critical component in any multidisciplinary treatment for patients with $\mathrm{MS}^{7,8}$

Current literature on sleep intervention in MS provide evidence in support of positive effects of pharmacological interventions on sleep, ${ }^{9}$ without any effect on fatigue, mood, and objective measures of sleep. ${ }^{10}$ Although nonpharmacological sleep interventions in MS such as cognitive-behavioral therapy (CBT) has received support as an effective and cost-effective therapy intervention leading to improved sleep quality, limitations exist in the current literature, such as studies only including female participants and failing to utilize clinical trials to test this intervention method. ${ }^{11,12}$ Physical activity and aerobic exercise have also received support and studies demonstrate a positive relationship with sleep parameters in patients with MS. ${ }^{13}$ However, these interventions produce different effects in the outcome measures of fatigue and depression. ${ }^{14}$ Additionally, educational interventions such as improving sleep hygiene behaviors have been recognized as effective methods to improve sleep quality. ${ }^{15}$ It is likely that disagreement and controversy exist when determining the most effective method of sleep interventions in MS as a result of the complex nature of this disease and its consequences, including tone disturbance, pain, cognitive disorder, and disability. Taken together, there is growing literature on the presence of sleep disorders in MS, although few intervention studies have been published and further research is warranted. $^{12,16}$

Evidence in the current literature supports the influence of sleep quality and severity of fatigue on the quality of life in patients with MS. ${ }^{17}$ Fatigue is a common symptom present in $\mathrm{MS}^{5}$ and has a bidirectional relationship with sleep quality. ${ }^{1,5,18,19}$ Several studies have highlighted the potential for interventions of sleep disorders to improve fatigue and vice versa. ${ }^{1,4,20}$ Additionally, since sleep quality has been recognized as a factor that affects the QOL of patients with MS, they frequently suffer from poorer health-related quality of life (HRQoL) $)^{4,21,22}$ and occupational engagement. ${ }^{23}$ In the present literature, the two factors of fatigue and quality of life can be considered to be functional outcomes when assessing the quality of sleep intervention programs. ${ }^{4}$

The inclusion of occupational therapy (OT) as part of a rehabilitation team can address many of the problems caused by $\mathrm{MS}^{24}$ Based on current evidence found in the literature, OT interventions for MS are primarily focused on remediation and compensatory approaches. Using remediative and compensatory approaches, OT interventions for MS are primarily focused on helping patients to be more independent in life. In remediative approaches, OT uses exercises to strengthen fine motor skills, coordination, mental alertness, and balance. Examples of OT interventions for patients with MS using a remediation approach are exercises that improve finger dexterity to enable tasks like buttoning a shirt and exercises that improve balance when standing and prevent falling. In a compensatory approach, OT interventions help improve performance skills by setting up adaptive devices at work, school, and home that would assist with routine daily activities and teach energy conservation to patients with MS. The use of wheelchairs, canes, and walkers to improve mobility, and the use of visual aids, like magnifying readers, are examples of OT interventions with a compensatory approach. ${ }^{25-27}$

In occupational therapy practice framework, rest/sleep is introduced as an occupation. Sleep has a critical impact on performance in other occupational domains such as education, ADL, work, play, leisure and social participation. ${ }^{28}$ Although the use of occupational therapy appears to be an essential intervention to address sleep disturbance, there is a lack of evidence documenting the effectiveness of this intervention in the current literature. According to the scoping review by Leland et al, sleep intervention based on OT in older adults has primarily been implemented in four intervention domains. These domains consisted of cognitive-behavioral therapy for insomnia, physical activity (such as resistance training, dancing, stretching, and running), multi-components 
interventions (environmental modifications such as noise and temperature), and other intervention strategies (such as light therapy and strategies to enhance the sleep environment). ${ }^{23}$

No occupational therapy programs for sleep-targeted interventions currently exist for patients with MS. Consequently, the first goal of this study was to design a sleep-targeted intervention program for MS and examine the efficacy of the intervention and compare it to traditional occupational therapy interventions using the primary outcome of sleep quality. The second goal follows the current literature $^{20}$ and we hypothesized that the improvement of sleep quality using our sleep-targeted intervention program would reduce the negative impact of fatigue in patients with MS. Our third goal follows previous literature $^{5,21,22}$ and led to our hypothesis that the quality of life of patients with MS will increase with the developed sleep-targeted intervention program. Therefore, this pilot randomized clinical trial study investigated sleep quality as the primary outcome and fatigue and quality of life as secondary outcomes of the sleep-targeted intervention program for MS.

\section{Patients and Methods Trial Design}

The current study was a pilot single-center, single-blinded (assessor-blind), parallel two-arm randomized-controlled trial with participants allocated on a 1:1 ratio to the intervention and control group. This clinical trial was conducted in accordance with the Declaration of Helsinki. It was approved by the ethical committee of the Iran University of Medical Sciences (IR.IUMS.REC 1395.9413355002) and was registered in the Iranian registry of clinical trials (IRCT20120910010806N6). The authors do not intend to share individual de-identified participant data at this time. All studied participants signed the written informed consent prior to enrollment.

\section{Participants}

The current study enrolled 20 participants refereed to Tehran rehabilitation centers who were diagnosed with MS according to the 2010 revision of the McDonald criteria for a minimum of 6 months and were confirmed by an expert neurologist. These patients ranged in age from 18 to 50 years with at least 5 years education, an Expanded Disability Status Score (EDSS) $<6$, and a MiniMental Status Examination (MMSE) score $>23$, since in the valid Persian version of MMSE the cutoff score 23 has been calculated as the best one. ${ }^{29}$ All participants had complaints of insomnia and poor sleep quality and scored $\geq 5$ on the Pittsburg sleep questionnaire indices ( $\mathrm{PSQI}^{30}$ ). Patients who reported diagnosed yet untreated sleep problems such as restlessness leg syndrome and a high likelihood of obstructive sleep apnea (OSA) based on the Berlin questionnaire were excluded. These patients with OSA were excluded since they may require specific interventions such as continuous positive airway pressure $(\mathrm{CPAP})^{2}$ and the interventions in this study were more chosen to be more effective for patients with insomnia. Furthermore, a similar study by Sater, Gudesblatt, KresaReahl, Brandes, and Sater $(2016)^{10}$ excluded these cases as a strategy to control for confounding variables. Additionally, patients with previous diagnoses of psychiatric disorders including mood disorders, anxiety disorders, and substance use disorders that interfere with normal sleep were excluded. All participants denied the use of hypnotic drugs. Figure 1 shows the flowchart of the current study.

\section{Setting}

The study was conducted in the occupational therapy clinic of Iran University of Medical Sciences (IUMS) located in Tehran, Iran. In this clinic, patients with different types of physical dysfunction, including patients with MS, are provided occupational therapy services. Patients are typically referred by physicians and receive an initial assessment before receiving a customized specific occupational therapy program for their presenting problems. A "quiet room" is located in the clinic and was utilized in the current study. The intervention sessions for both groups were implemented between 8:00 am and 1:00 pm.

\section{Randomization, Allocation Concealment, and Blinding}

The blinded assessor evaluated 20 patients with MS who met the inclusion criteria using Pittsburgh Sleep Quality Index (PSQI) the day before starting the intervention procedure. Each patient's PSQI score was stratified to ensure that the severity of sleep disturbances contributed similar numbers of participants to each group. The participants were then assigned to one of the two groups in the study, patients receiving care-as-usual for MS (CAU) or patients receiving care-as-usual plus intervention (CAU + Intervention), using a random number generator and sealed opaque envelopes. An 


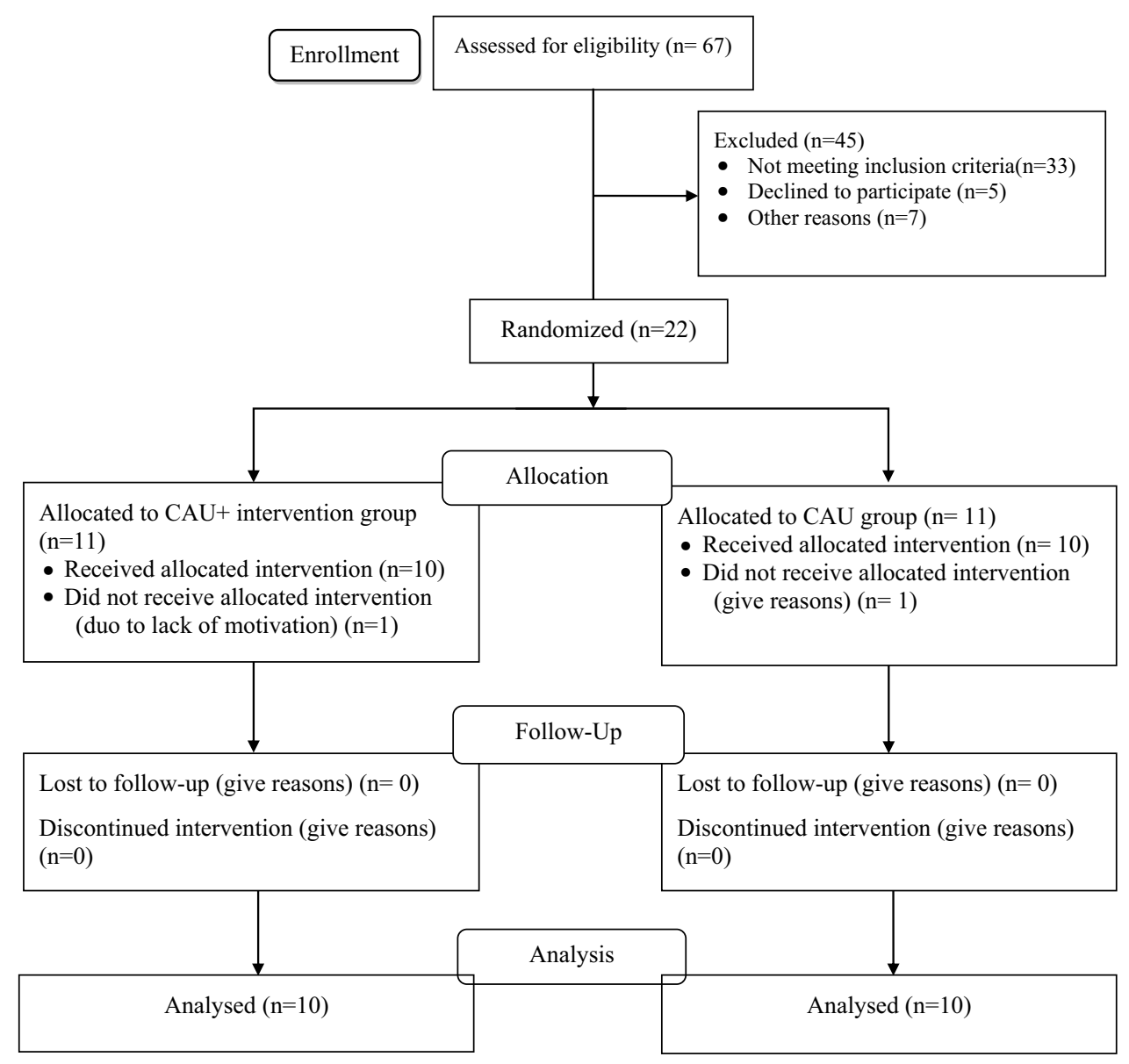

$\mathrm{CAU}=$ Care-as-usual.

Figure I Flowchart utilized in the study.

Abbreviation: CAU, care-as-usual.

independent individual who was blinded to the study aims conducted the number generation for the group allocation and concealed the allocation using the sealed opaque envelopes.

\section{Procedures}

After the Ethics Committee of Iran University of Medical Sciences (IUMS) approved the study design, the blinded assessor evaluated 20 patients with MS who met the inclusion criteria using the Persian version of Pittsburgh Sleep Quality Index (PSQI) Fatigue Impact Scale (FIS), ${ }^{31}$ Fatigue Severity Scale (FSS), and Short-Form Health Survey (SF-36), in a random order the day before starting intervention. After two groups were assigned, one occupational therapist who had 5 years of work experience with patients with MS was chosen randomly and assigned to each group. The therapists were unaware of the aims of the study and intervention specifics in the opposing group.
Taking into consideration the specific symptoms of patients with MS (low fatigue threshold, restrictions on attending a clinic, and low endurance), the intervention program was designed to be as short as possible and contain the least number of sessions. Therefore, both groups underwent an intervention program that included meeting once a week for 30-45 minutes for 8 sessions in an 8-month period from April to November 2018. All participants received 2-3 sessions per week by telephone, in order to address any issues that arose when executing all parts of the session's agenda. All participants received a workbook in the first session in which the therapist noted the comments and exercises to be implemented in each session. Pre-tests were administered the day before the first session and post-tests were administered the day after the last session was completed.

\section{Interventions}

The intervention programs in each group were as follows: 
Patients receive care-as-usual plus intervention (CAU + Intervention): Since, currently, no evidence exists to support an occupational therapy-based intervention for sleep problems in patients with MS, this intervention was designed according to a review of the literature, with careful consideration given to the scoping review by Leland et $\mathrm{a}^{23}$ (2014) and Ho et $\mathrm{al}^{32}$ (2018) on the role of occupational therapists in the treatment of sleep problems. The intervention used in the current study was compromised of a combination of common interventions used for patients with sleep problems, including patients with MS and those receiving occupational therapy interventions. The common intervention included the interventions which effectiveness have been demonstrated in previous studies in patients with MS such as sleep hygiene education $^{13}$ (including an explanation of caffeine intake reduction, excessive night-time eating, and smoking cessation), physical activity ${ }^{14}$ (including moderate levels of aerobic exercises during the week lasting 10 to 30 minutes, depending on patient endurance, such as walking, running, and cycling four hours before falling asleep), $\mathrm{CBT}^{12}$ (including the exploration of dysfunctional thinking patterns surrounding sleep and teaching Jacobson relaxation techniques). Occupational therapy interventions were focused on the occupational balance based on the Person-EnvironmentOccupation Performance (PEOP) model. Accordingly, an occupational schedule was provided for participants to rearrange daytime activities to ensure time management for daily occupation and allow for participation in every occupation without fatigue. Environmental modifications, such as changes to a bedroom (including modifying noise, light, temperature, bedding, and technology use while in bed) was considered along with the occupational schedule. The schedule was associated with lifestyle modification which has gained support for improvement in quality of life. ${ }^{32}$ It should be noted that both sleep intervention and occupational therapy intervention were used together, potentially leading to an overlap in some areas. This intervention program is illustrated in Table 1. In the present study, the details of the program were explained to the patients during the first session which included extensive training, and the necessary follow-up was performed during the intervention period through telephone counseling.

Patients receiving care-as-usual (CAU): The program of this group consisted of exercises focused on strengthening fine motor skills, coordination, and balance, cognitive rehabilitation focused on memory and assessing ADL performance at home. Additionally, this included the prescription and training in the use of proper accessory equipment in the individual's house.

\section{Measures}

We used Persian versions of all measures used in this study. The versions were translated was performed according to the World Health Organization (WHO) process of translation and adaptation of instruments, which included forward and backward translations. In this process measures were initially forward-translated by 2 translators for cultural and linguistic modification and then backtranslated to English. The psychometric properties of all measures utilized in this study are described in further detail below.

\section{Pittsburgh Sleep Quality Index}

This questionnaire assesses sleep quality and disturbances over a 1-month interval using 19 questions based on 7 components of subjective sleep quality, sleep latency, sleep duration, habitual sleep efficiency, sleep disturbances, use of sleep medication, and daytime dysfunction. Each question ranges in score from 0 to 3 and the score of each component can be up to a maximum of 3 . The sum of these 7 component scores determines the overall score, which ranges from 0 to 21. Higher scores indicate lower sleep quality and a score higher than 5 indicates poor quality of sleep. ${ }^{33}$ The PSQI was translated into Persian and acceptable psychometric properties of the questionnaire have been reported by Farrahi Moghaddam et al (2014). ${ }^{30}$ These authors reported reliability of the Pittsburgh Index using the Cronbachacoefficient 0.77 for all subjects in their study, with 0.52 for the patient group and 0.78 for the control group. They also reported sensitivity and specificity of $94 \%$ and $72 \%$ for discrimination of insomniac patients from control group for this questionnaire. ${ }^{28}$

\section{Fatigue Impact Scale (FIS)}

The FIS was used to assess the impact of fatigue on everyday life activities. This scale includes 40 items in three subgroups: (1) Cognitive, which contains 10 items and focuses on the concentration, memory, thinking and organization of thoughts; (2) Physical, which includes 10 items and reflects motivation, endeavor, tolerance and coordination; and (3) Social, which includes 20 items and describes the effects of fatigue on isolation, emotion, stress, and communication. The intraclass correlation (ICC) values for inter-rater reliability in the physical, cognitive, and social subgroups of FIS and the whole questionnaire were $0.89,0.86,0.95$ and 0.98 , and for test-retest reliability were 
Table I The Program of Sleep Intervention Based on OT

\begin{tabular}{|c|c|}
\hline Sessions & Interventions \\
\hline First & $\begin{array}{l}\text { Education regarding sleep disorders. } \\
\text { Providing an occupation profile of sleep and daytime. Ask to take photo of their bedroom and restroom and explain in detail about the } \\
\text { temperature, noise, and light of bedroom for next session. A nutritionist was invited to explain to patients how to properly record } \\
\text { a nutrition table. Each patient was tasked with recording a weekly Nutrition Table for the next session. Patients were encouraged to } \\
\text { engage in } 10 \text { to } 30 \text { minutes (based on their endurance) of a moderate level aerobic exercise such as walking, running, or cycling with } \\
\text { moderate intensity during the week that occurred four hours before falling asleep. }\end{array}$ \\
\hline Second & $\begin{array}{l}\text { Set daily schedule based on the occupational profile. } \\
\text { Environmental modification: Modifying the environment, including noise, light, temperature, bedding, and technology use while in bed. } \\
\text { Address performance deficits or barriers to activities of daily living, particularly for bed mobility and toileting. } \\
\text { Teach cognitive-behavioral techniques, such as leaving the bedroom if awake and returning only when sleepy, or exploring self-talk } \\
\text { statements and teaching Jacobson relaxation techniques. }\end{array}$ \\
\hline Third & $\begin{array}{l}\text { Encourage health management behaviors such as smoking cessation, reduced caffeine intake, eating too much at nights, adopting } \\
\text { a balanced diet, and engaging in adequate exercise. } \\
\text { Teach cognitive behavioral techniques such as modifying environment, controlling disturbing stimulants, nutrition consultation, and } \\
\text { arranging sleep time. } \\
\text { Teach relaxation, strengthening, and stretching techniques in the muscles of the scapula, hamstring, and gastrocnemius. Perform } \\
\text { equilibrium and strengthening activities. }\end{array}$ \\
\hline Fourth & $\begin{array}{l}\text { Discuss the occupational balance and keeping busy during the day. } \\
\text { Teach cognitive behavioral strategies involving coping skills, stress management, and time management for doing occupations and } \\
\text { keeping balance among them. } \\
\text { Perform relaxation; doing equilibrium and strengthening activities. }\end{array}$ \\
\hline Fifth & Review cognitive behavioral exercises, modification, and discuss activities of daily living and home modification. \\
\hline Sixth & Physical exercise as explained in the first session and ask about the balance of occupations. \\
\hline Seventh & The last modifications and final consultations \\
\hline Eighth & Review all exercises, trainings, and final advice. Receive feedback. \\
\hline
\end{tabular}

$0.86,0.78,0.92$ and 0.93 , respectively. The above findings taken together with a Cronbach $\alpha$ of 0.95 indicate a high reliability for the FIS Persian version. ${ }^{31}$

\section{Fatigue Severity Scale (FSS)}

The FSS is comprised of 9 items. Patients report their feelings during the past two weeks for each item, ranging from 1 to 7 points. A low score indicates a high disagreement with the items, while a high score shows strong agreement with the items, and the total score is in the range of 9-63. The internal consistency of the items in the questionnaire using Cronbach's alpha coefficient was 0.96 . The correlation of each item with another item was 0.4 . The ICC coefficient was $0.93 .^{34}$

\section{Short-Form Health Survey (SF-36)}

The SF-36 questionnaire consists of 2 main subgroups of physical and mental health, with each subgroup including 4 domains which generally study 8 areas. Physical areas include physical function (10 items), role limitation (4 items), body pain (7 items), and general health (4 items). Furthermore, mental health areas include social function ( 2 items), role emotion ( 3 items), vitality ( 4 items), and mental health ( 5 items). Scores for the SF-36 scales range between 0 and 100, with higher scores indicating a better health-related quality of life. ${ }^{35}$ In the present study, a Persian version of the SF-36 was used. The Internal consistency analysis of Persian version of SF-36 ranged from 0.77 to 0.90 except for the area of vitality. The correlation coefficient is greater than the recommended value of 0.4 (range of coefficients is 58.8 to 0.95 ). This survey is currently the most widely used instrument for measuring the quality of life in the world. ${ }^{36}$

\section{Mini-Mental State Examination (MMSE)}

The MMSE, or Folstein test, is a brief 30-point questionnaire that is used to screen for cognitive impairment. It is commonly used in medicine to screen for dementia. It is 
also used to estimate the severity of cognitive impairment and to follow the course of cognitive changes in an individual over time, thus making it an effective way to document an individual's response to treatment. It takes approximately 10 minutes to complete and samples functions involving arithmetic, memory, and orientation. This test is not a mental status examination. ${ }^{37}$ This measure was translated into Persian and it has shown good construct validity, high test-retest and inter-rater reliability. ${ }^{29}$ It should be noted that there may be other gold-standard measures for the assessment of cognitive impairment, but due to not having access to valid Persian versions of other assessments; the MMSE was chosen for use in the present study.

\section{Statistical Analysis}

Data were analyzed using SPSS, version 16.0 (SPSS Inc., Chicago, IL, USA). The Mann-Whitney $U$-test and the Wilcoxon Signed Ranks Test were used for comparisons of non-parametrically variables of PSQI, FIS, and SF-36. The total scores of PSQI, FIS, FSS, and SF-36 were normally distributed. Independent sample t-tests and paired t-tests were utilized to compare variables in the two groups, CAU and CAU plus intervention.

The level of significance was $\mathrm{p}=0.05$ and was adjusted familywise for multiple comparisons by the Error-Rates-Method and significant differences between the test and control groups were identified. The Cohen's $\mathrm{d}$ was calculated, with 0.20 indicating a small effect, 0.50 indicating a medium effect, and 0.80 indicating a large effect. $^{38}$

\section{Results}

The results of 20 patients with MS who completed the intervention programs of present study were analyzed. As shown in Table 2, the CAU plus intervention group (2 males and 8 females) and CAU group ( 2 males and 8 females) were not significantly different in terms of sex, age, EDSS and pretest scores of the PSQI $(\mathrm{P}>0.50)$.

The sleep quality in the CAU plus intervention group (mean $\pm \mathrm{SD}=7.2 \pm 1.03$ ) significantly improved $(\mathrm{p}<0.001)$; however, in the CAU group (mean $\pm \mathrm{SD}=$ $13.20 \pm 3.48)$, the sleep quality did not improve. The results of independent sample t-tests revealed a significant difference $(\mathrm{T}(10.56)=-5.21, p<0.001$, effect size $=0.60)$ in total PSQI scores. However, two groups in two PSQI subscales, sleep efficacy and sleep medication, did not have any significant differences (Table 3 ).
Table 2 The Mean (SD) of Character of Participants by CAU and $\mathrm{CAU}+$ Intervention Group at Baseline

\begin{tabular}{|l|l|l|l|}
\hline \multirow{2}{*}{ Characteristics } & CAU & CAU+Interv. & \multirow{2}{*}{ P value } \\
\cline { 2 - 3 } & Mean (SD) & Mean (SD) & \\
\hline Age & $39.7(7.90)$ & $37.5(8.89)$ & $0.57^{* *}$ \\
EDSS & $1.2(1.03)$ & $1.6(0.97)$ & $0.38^{* *}$ \\
PSQI & $12.8(2.86)$ & $15.2(2.44)$ & $0.06 * *$ \\
SSQ & $2.3(0.48)$ & $2.3(0.48)$ & $0.66^{*}$ \\
SL & $2.5(0.71)$ & $2.5(0.71)$ & $1 *$ \\
SDU & $2.1(0.74)$ & $2.1(0.74)$ & $0.18^{*}$ \\
SE & $2(0.94)$ & $2(0.94)$ & $0.16^{*}$ \\
SDB & $1.7(0.67)$ & $1.7(0.67)$ & $0.33^{*}$ \\
USM & $0.4(0.96)$ & $0.4(0.96)$ & $0.19 *$ \\
DD & $1.8(0.79)$ & $1.8(0.79)$ & $0.27^{*}$ \\
FIS & $80(18.93)$ & $97(15.53)$ & $0.04^{* *}$ \\
FSS & $5.98(0.86)$ & $6.26(0.79)$ & $0.46^{* *}$ \\
PCS & $159.5(50.1)$ & $148.3(37.13)$ & $0.58^{* *}$ \\
MCS & $162.4(28.6)$ & $150.5(29.04)$ & $0.37^{* *}$ \\
\hline
\end{tabular}

As each of the two groups had significant differences in baseline scores of the FIS, the gain difference (post-test score compared to baseline score) was calculated to compare these secondary outcomes of the interventions. The gain difference of fatigue impact (by FIS) decreased more in the CAU plus intervention group (mean $\pm \mathrm{SD}=-43.30$ \pm 15.86 ) than the CAU group (mean $\pm \mathrm{SD}=7.4 \pm 7.41$ ) and the impact of fatigue decreased in both groups (Table 3). The results of independent sample t-tests revealed this difference was significant $(\mathrm{T}(18)=-9.16$, $p<0.001$, effect size $=0.82$ ). The comparison of subscales is shown in Table 3.

The gain difference of the Fatigue Severity Scale (FSS) decreased in the CAU plus intervention group (mean $\pm \mathrm{SD}=$ - $14.30 \pm 5.80$ ) compared to the CAU group (mean $\pm \mathrm{SD}=4.90$ $\pm 5.44)$ and in both groups, the severity of fatigue decreased (Table 3). The results of independent sample t-tests revealed this difference was significant $(\mathrm{T}(18)=-7.61, \mathrm{p}<0.001$, effect size $=0.76$ ). The comparison of subscales is shown in Table 3 . The scores of physical and mental components of SF-36 improved significantly only in the CAU plus intervention group ( $p>0.001$ ). The results of the Mann-Whitney $U$-test revealed a significant difference between the two groups in all subscales of the SF-36 except the social subscale (Table 3).

\section{Discussion}

In this pilot clinical trial study, the effects of a sleeptargeted occupational therapy intervention on sleep quality, fatigue, and quality of life in patients with MS was 
Table 3 The Within-Group and Between-Group Analysis of the Pittsburgh Sleep Quality Index, Fatigue Impact Scale, Fatigue Severity Scale, and SF-36 Scores in Two Groups, CAU Group $(n=10)$ and CAU + Intervention Group $(n=10)$

\begin{tabular}{|c|c|c|c|c|c|c|c|c|c|c|}
\hline \multicolumn{2}{|c|}{ Variables } & \multicolumn{2}{|l|}{ CAU Group } & \multirow{2}{*}{$\begin{array}{l}\text { Within- } \\
\text { Group P }\end{array}$} & \multicolumn{2}{|c|}{ CAU+Interv. Group } & \multirow{2}{*}{$\begin{array}{l}\text { Within- } \\
\text { Group P }\end{array}$} & \multicolumn{3}{|c|}{ Between Groups After Treatment } \\
\hline & & $\begin{array}{l}\text { Baseline } \\
\text { Mean (SD) }\end{array}$ & $\begin{array}{l}\text { Outcome } \\
\text { Mean (SD) }\end{array}$ & & $\begin{array}{l}\text { Baseline } \\
\text { Mean (SD) }\end{array}$ & $\begin{array}{l}\text { Outcome } \\
\text { Mean (SD) }\end{array}$ & & $\begin{array}{l}\text { Mean } \\
\text { Difference } \\
\text { (SE) }\end{array}$ & $P$ value & $\begin{array}{l}\text { Effect } \\
\text { Size }\end{array}$ \\
\hline PSQI & $\begin{array}{l}\text { SSQ } \\
\text { SL } \\
\text { SDU } \\
\text { SE } \\
\text { SDB } \\
\text { USM } \\
\text { DD } \\
\text { Total }\end{array}$ & $\begin{array}{l}2.3(0.48) \\
2.5(0.7 I) \\
2.1(0.74) \\
2(0.94) \\
1.7(0.67) \\
0.4(0.96) \\
1.8(0.79) \\
12.8(2.85)\end{array}$ & $\begin{array}{l}2.3(0.48) \\
2.6(0.70) \\
2.1(0.74) \\
2(0.94) \\
2.1(0.73) \\
0(0) \\
2.1(0.74) \\
13.2(3.48)\end{array}$ & $\begin{array}{l}I^{*} \\
0.317^{*} \\
I^{*} \\
I^{*} \\
0.206^{*} \\
0.180^{*} \\
0.317^{*} \\
0.42^{* *}\end{array}$ & $\begin{array}{l}2.4(0.52) \\
2.5(0.97) \\
2.5(0.53) \\
2.5(0.52) \\
2(0.67) \\
1.1(1.23) \\
2.2(0.79) \\
15.2(2.44)\end{array}$ & $\begin{array}{l}I(0) \\
I .4(0.70) \\
I .2(0.42) \\
I .4(0.52) \\
I .2(0.63) \\
0(0) \\
0.9(0.32) \\
7.2(1.03)\end{array}$ & $\begin{array}{l}0.004^{*} \\
0.009 * \\
0.004^{*} \\
0.005^{*} \\
0.023^{*} \\
0.024 * \\
0.006 * \\
<0.00 \text { I* }\end{array}$ & $\begin{array}{l}-1.3(0.15) \\
-1.2(0.31) \\
-0.9(0.27) \\
-0.6(0.34) \\
-0.9(0.31) \\
0(0) \\
-1.2(0.25) \\
-6(1.15)\end{array}$ & $\begin{array}{l}<0.00 I^{* * * *} \\
0.003^{* * *} \\
0.01 I^{* * * *} \\
0.19^{* * * *} \\
0.02^{* * * *} \\
I^{* * *} \\
0.00 I^{* * *} \\
<0.001^{* * * *}\end{array}$ & $\begin{array}{l}0.90 \\
0.48 \\
0.39 \\
- \\
0.32 \\
- \\
0.61 \\
0.60\end{array}$ \\
\hline FIS & $\begin{array}{l}\text { CFI } \\
\text { PFI } \\
\text { SFI } \\
\text { Total }\end{array}$ & $\begin{array}{l}13.5(7.2) \\
27.4(2.99) \\
39.3(10.11) \\
80.2(18.94)\end{array}$ & $\begin{array}{l}14.8(7.61) \\
29.9(4.36) \\
42.9(\mid 2.6) \\
87.6(21.52)\end{array}$ & $\begin{array}{l}0.041^{* *} \\
0.059^{*} \\
0.050^{*} \\
0.012^{* *}\end{array}$ & $\begin{array}{l}20.5(5.97) \\
29.3(5.33) \\
47.2(9.49) \\
97(15.53)\end{array}$ & $\begin{array}{l}11.5(4.24) \\
17.6(4.94) \\
24.6(8.40) \\
53.7(13.57)\end{array}$ & $\begin{array}{l}0.008^{*} \\
0.005^{*} \\
0.005^{*} \\
<0.00 I^{* *}\end{array}$ & $\begin{array}{l}-13.3(2.8) \\
-12.3(2.1) \\
-18.3(4.8) \\
-33.9(8.04)\end{array}$ & $\begin{array}{l}<0.00 I^{* * * *} \\
<0.00 I^{* * *} \\
<0.00 I^{* * * *} \\
<0.001^{* * * * *}\end{array}$ & $\begin{array}{l}0.68 \\
0.76 \\
0.72 \\
0.82\end{array}$ \\
\hline FSS & Total & $5.97(0.86)$ & $6.52(0.46)$ & $0.018 * *$ & $6.3(0.79)$ & $4.67(1.05)$ & $0.005 * *$ & $-1.85(0.36)$ & $<0.001 * * * *$ & 0.76 \\
\hline SF-36 & $\begin{array}{l}\text { PF } \\
\text { RP } \\
\text { RE } \\
\text { VT } \\
\text { MH } \\
\text { SF } \\
\text { BP } \\
\text { GH }\end{array}$ & $\begin{array}{l}50(21.86) \\
25(23.57) \\
20(17.21) \\
38(7.89) \\
54.4(10.19) \\
50(10.21) \\
52(16.24) \\
32.49(12.2)\end{array}$ & $\begin{array}{l}44.5(\mid 8.92) \\
15(\mid 7.4) \\
10(\mid 6.1) \\
35.5(8.32) \\
56.4(\mid 1.1) \\
50(\mid 0.2) \\
52(\mid 6.3) \\
32.5(\mid 2.2)\end{array}$ & $\begin{array}{l}0.026^{*} \\
0.414^{*} \\
0.083^{*} \\
0.096^{*} \\
0.518^{*} \\
I^{*} \\
0.317^{*} \\
0.317^{*}\end{array}$ & $\begin{array}{l}50.5(21.92) \\
12.5(17.68) \\
6.67(14.05) \\
36(9.66) \\
50.33(12.9) \\
57.5(13.44) \\
50.8(12.42) \\
34.6(11.46)\end{array}$ & $\begin{array}{l}76(16.12) \\
55(34.9) \\
76.7(16.11) \\
53.3(8.82) \\
70.8(8.65) \\
65(14.2) \\
65(11.1) \\
45.4(16.84)\end{array}$ & $\begin{array}{l}0.005^{*} \\
0.241^{*} \\
0.005^{*} \\
0.005^{*} \\
0.005^{*} \\
0.084^{*} \\
0.018^{*} \\
0.035^{*}\end{array}$ & $\begin{array}{l}31.5(7.86) \\
40(\mid 2.4) \\
66.65(7.2) \\
17.75(3.83) \\
14.4(4.44) \\
15(5.52) \\
13.05(6.22) \\
12.92(6.58)\end{array}$ & $\begin{array}{l}<0.00 I^{* * * *} \\
0.00 I^{* * *} \\
<0.00 I^{* * * *} \\
<0.001^{* * *} \\
0.00 I^{* * * *} \\
0.143^{* * *} \\
0.004^{* * *} \\
0.02^{* * *}\end{array}$ & $\begin{array}{l}0.76 \\
0.61 \\
0.80 \\
0.76 \\
0.58 \\
- \\
0.51 \\
0.34\end{array}$ \\
\hline $\begin{array}{l}\text { SF } \\
\text { Sum. }\end{array}$ & $\begin{array}{l}\text { PCS } \\
\text { MCS }\end{array}$ & $\begin{array}{l}159.5(50.1) \\
162.4(28.6)\end{array}$ & $\begin{array}{l}143.9(48.3) \\
151.9(24.9)\end{array}$ & $\begin{array}{l}0.087 * * \\
0.174 * *\end{array}$ & $\begin{array}{l}148.3(37.13) \\
150.5(29.04)\end{array}$ & $\begin{array}{l}24 I .4 I(38 . I) \\
265.7(35.9)\end{array}$ & $\begin{array}{l}<0.001 * * \\
<0.001 * *\end{array}$ & $\begin{array}{l}I \mid 3.8(\mid 3.9) \\
97.47(\mid 9.4)\end{array}$ & $\begin{array}{l}<0.001^{* * * * * *} \\
<0.001^{* * * * *}\end{array}$ & $\begin{array}{l}0.67 \\
0.82\end{array}$ \\
\hline
\end{tabular}

Notes: Data were presented as mean (standard deviation) and mean difference (Std. Error Difference). *Wilcoxon Signed Ranks Test; **Paired t-test; ***Mann-Whitney U; *****independent T.

Abbreviations: PSQI, The Pittsburgh Sleep Quality Index; SSQ, subjective sleep quality; SL, sleep latency; SDU, sleep duration; SE, sleep efficiency; SDB, sleep disturbance; USM, use of sleep medication; DD, daytime dysfunction; FIS, Fatigue Impact Scale; CFI, cognitive part of Fatigue Impact Scale; PFI, physical part of Fatigue Impact Scale; SFI, social part of Fatigue Impact Scale; FSS, fatigue Severity Scale; SF-36, The Short Form (36) Health Survey; PF, physical functioning; RP, role limitations due to physical health; RE, role limitations due to emotional problems; VT, energy/fatigue; $\mathrm{MH}$, emotional well-being; SF, social functioning; BP, bodily pain; GH, general health; PCS, physical health component; MCS, mental health component score; CAU, care-as-usual in occupational therapy; CAU+ Interv., care-as-usual in occupational therapy + sleep intervention; SF-36 Sum, SF36 summary.

compared to a control group who received a typical occupational therapy routine program for MS. The results showed a moderate to large effect size of this sleep intervention program on total scores of PSQI and large effect size in subjective quality of sleep subgroup of PSQI based on Cohen's d effect sizes. It is worth noting that this program yielded positive outcomes for patients with MS in all three areas as defined by an improvement in sleep quality (the study's primary outcome), reduction in fatigue (the secondary outcome), and enhancing the individual's quality of life (the third outcome). The positive effects of exercise, CBT, or educational interventions in the improvement of sleep disorders in patients with MS has been reported in the previous studies. ${ }^{10,12,14}$ It is reported that exercise is associated with changes in endocrine system functioning, as well as reducing depression and anxiety levels that lead to an improvement in quality of sleep. ${ }^{13}$ Additionally, the effectiveness of CBT has been attributed to changes in thoughts and behaviors related to sleep which may maintain insomnia. ${ }^{12,14}$

As previously stated, the intervention utilized in the present study was based on the PEOP model ${ }^{32}$ and focuses on three levels: person, environment, and occupation. In the sleep-targeted program, which is occupation-based, all of these aspects were considered and incorporated into the program. Regarding the first level, the individual person, both the physical (spasticity and weakness) and mental (thinking and mind) aspects of the human body were 
considered and targeted using CBT, aerobic, and relaxation exercises. The second factor, the environment, is addressed in treatment with the inclusion of accommodating and rearranging the patient's bedroom in order to eliminate disturbances. This structure can involve both physical and mental aspect of a given patients while she/he try to modify environment. Furthermore, this intervention is considered as a purposeful and meaningful activity for the patient to help him/herself. According to OT practice framework, meaningful activity can develop health in individuals. ${ }^{28}$ It should be noted that it is in consistent with perceived sense of control over one's symptoms seen in patients' with insomnia who participated CBT program. ${ }^{12}$ The final factor, occupation, was addressed by restructuring daytime activity and establishing occupational balance. ${ }^{28}$ Occupational balance is another concept in OT practice framework which emphasizes the importance of balance among people's occupations including work, play, and self-care. Accordingly, the balance can lead to healthy behavior and improved occupation performance. In this study, the restructuring of daytime activity despite physical limitation and engagement in all aspects of occupation may result in a reduction of stress and a sense of better health. ${ }^{32}$ Therefore, the intervention can be considered to be effective from an OT perspective.

Results of this study show that the proposed sleep intervention had a significant effect on decreasing fatigue and increasing the quality of life of the patients with MS (the study's secondary outcome). This pilot study utilized Cohen's d to identify effect size and found a large effect size of the sleep intervention program based on total scores of FIS, moderate to large effect size based on total scores of FSS, cognitive, physical, and social aspects of FIS. Fatigue was reduced in both severity and impact on life domains in the sleep-targeted occupational therapy intervention group. Due to the decision that the sleep intervention program considered the most common factors of sleep and fatigue such as physical, cognitive-emotional aspects, environmental modification, set daily schedule, and occupational balance, decreasing fatigue and sleep improvement was expected. Although the large body of current literature confirms the bidirectional relationship between sleep disorders and increasing fatigue, there is controversy regarding the result of some of these clinical trial studies. In a study conducted by Sadeghi Bahmani et al, the physical activity intervention improved sleep without any effect on fatigue. ${ }^{14}$ However, in a study conducted by Dayapoglu et al, the Progressive Muscle Relaxation Technique intervention decreased patients' fatigue level and improved their sleep quality. ${ }^{39}$ These findings suggest that this discrepancy is due to the multifactorial nature of sleep disturbances and fatigue in patients with MS. ${ }^{40}$

Another secondary outcome in this study was quality of life, which was improved in the sleep target occupational therapy group with moderate to large effect sizes in all aspects except in the general health subgroup, which had a small effect size based on Cohen's d. However, quality of life in the social function aspect showed no differences between the two groups. The results of several previously published studies indicate that sleep disorders can reduce health-related quality of life in patients with MS and should be considered as an important confounder in all studies investigating health-related quality of life in patients with MS. ${ }^{41}$ Wulff et al proposed that physiological changes in cognitive and emotional functioning in patients with MS and other neurodegenerative diseases act as a common mechanism that often leads to the development of sleep disorders. ${ }^{42}$ The improvement of quality of life in the domains of physical, emotional functioning, fatigue reduction, and pain reduction in the present study may be due to the use of exercise and CBT interventions. These interventions have the potential to reduce signs of tension, contribute to better sleep quality, and increase relaxation. Conversely, occupational balance and an increase in resilience can lead to a feeling of increased efficiency. Therefore, improvement in sleep quality plays an important role in both an individual's autonomy the quality of life in patients placed in the intervention group.

\section{Strengths and Limitations}

As the first study to investigate the effects of a sleeptargeted intervention that incorporates occupational therapy in patients with MS, the study was seen in light both strengths and limitations. Regarding the current study's strengths, the intervention was administered simultaneously with occupational therapy interventions. This provides a good opportunity for patients to receive intervention for both sleep and physical problems that in turn saves time for both patient and their caregivers. Secondly, the intervention is fairly easy and flexible and can be conducted by telephone and virtual context, especially for patients with limited access to occupational therapy clinics. Finally, by improving sleep quality and reducing fatigue, patients are more motivated to participate in rehabilitation programs that ultimately lead to better rehabilitation outcomes.

The present study also had limitations that should be addressed. The first limitation was a small sample size that 
could potentially lead to inaccurate generalizations of the results, suggesting that caution should be used when generalizing these findings to other populations that significantly differ from the participants studied. Secondly, the study was limited through the inability to assess sleep quality using objective measures such as polysomnography in order to more accurately determine the efficacy of our intention. Third, as recommended in OT literature, ${ }^{22}$ the intervention used in this study was a combination of several strategies such as sleep hygiene and physical exercise, and we were therefore unable to clearly identify which component had the highest level of influence on the outcomes. Separating these components in future studies can lead to more precise results for occupational therapists who may want to utilize these interventions. Finally, a long-term follow-up was not utilized to evaluate the long-term effects of the intervention.

\section{Implications}

The present study has implications for both clinical practice and aspects of future research. The results of this study should inform occupational therapists who work with patients diagnosed with MS and use interventions aimed at improving sleep quality, reducing fatigue, and ultimately improving overall quality of life. Furthermore, it can be modified and utilized for patients with progressive neurological disorders. It is also recommended that academic researchers pursuing research in this area conduct a study with a larger sample size in order to increase the generalizability of the results. Lastly, future studies should consider the incorporation of a long-term follow-up with the patients who received the intervention as well as the use of objective measures of sleep quality assessment.

\section{Conclusion}

The present study demonstrated that the examined intervention program can improve sleep quality and quality of life while decreasing fatigue in patients with MS. Adjunction of this program, if results are similar with more patients, to routine occupational therapy (OT) interventions can help improve the rehabilitation program of MS patients.

\section{Acknowledgments}

The authors wish to thank all who participated in this study.

\section{Disclosure}

The authors report no conflicts of interest in this work.

\section{References}

1. Kister I, Bacon TE, Chamot E, et al. Natural history of multiple sclerosis symptoms. Int J MS Care. 2013;15(3):146-158. doi:10. 7224/1537-2073.2012-053

2. Sakkas GK, Giannaki CD, Karatzaferi C, Manconi M. Sleep abnormalities in multiple sclerosis. Curr Treat Options Neurol. 2019;21(1):4. doi:10.1007/s11940-019-0544-7

3. Brass SD, Duquette P, Proulx-Therrien J, Auerbach S. Sleep disorders in patients with multiple sclerosis. Sleep Med Rev. 2010;14 (2):121-129. doi:10.1016/j.smrv.2009.07.005

4. Kotterba S, Neusser T, Norenberg C, et al. Sleep quality, daytime sleepiness, fatigue, and quality of life in patients with multiple sclerosis treated with interferon beta- $1 \mathrm{~b}$ : results from a prospective observational cohort study. BMC Neurol. 2018;18(1):123. doi:10.1186/s12883-018-1113-5

5. Krupp L. Fatigue is intrinsic to multiple sclerosis (MS) and is the most commonly reported symptom of the disease. Mult Scler. 2006;12(4):367-368. doi:10.1191/135248506ms1373ed

6. Rezaie L, Khazaie H, Yazdani F. Exploration of the experience of living with chronic insomnia: a qualitative study. Sleep Sci. 2016;9 (3):179-185. doi:10.1016/j.slsci.2016.07.001

7. Caminero A, Bartolome M. Sleep disturbances in multiple sclerosis. J Neurol Sci. 2011;309(1-2):86-91. doi:10.1016/j.jns.2011.07.015

8. Garland SN, Scurrey SRM, Ploughman M. Factors associated with poor sleep in older adults with multiple sclerosis. Int J Behav Med. 2017;24(6):937-945. doi:10.1007/s12529-017-9653-4

9. Morris G, Stubbs B, Kohler CA, et al. The putative role of oxidative stress and inflammation in the pathophysiology of sleep dysfunction across neuropsychiatric disorders: focus on chronic fatigue syndrome, bipolar disorder and multiple sclerosis. Sleep Med Rev. 2018;41:255-265. doi:10.1016/j.smrv.2018.03.007

10. Sater RA, Gudesblatt M, Kresa-Reahl K, Brandes DW, Sater P. NAPS-MS: natalizumab effects on parameters of sleep in patients with multiple sclerosis. Int J MS Care. 2016;18(4):177-182. doi:10.7224/1537-2073.2015-033

11. Abbasi SM, Alimohammadi NP, Pahlavanzadeh SM. Effectiveness of cognitive behavioral therapy on the quality of sleep in women with multiple sclerosis: a randomized controlled trial study. Int J Community Based Nurs Midwifery. 2016;4(4):320-328.

12. Clancy M, Drerup M, Sullivan AB. Outcomes of cognitive-behavioral treatment for insomnia on insomnia, depression, and fatigue for individuals with multiple sclerosis: a case series. Int J MS Care. 2015;17 (6):261-267. doi:10.7224/1537-2073.2014-071

13. Aburub A, Khalil H, Al-Sharman A, Alomari M, Khabour O. The association between physical activity and sleep characteristics in people with multiple sclerosis. Mult Scler Relat Disord. 2017;12:29-33. doi:10.1016/j.msard.2016.12.010

14. Sadeghi Bahmani D, Razazian N, Farnia V, Alikhani M, Tatari F, Brand S. Compared to an active control condition, in persons with multiple sclerosis two different types of exercise training improved sleep and depression, but not fatigue, paresthesia, and intolerance of uncertainty. Mult Scler Relat Disord. 2019;36:101356. doi:10.1016/j.msard.2019.07.032

15. Newland P, Lorenz RA, Smith JM, Dean E, Newland J, Cavazos P. The relationship among multiple sclerosis-related symptoms, sleep quality, and sleep hygiene behaviors. J Neurosci Nurs. 2019;51 (1):37-42. doi:10.1097/JNN.0000000000000409

16. Lanza G, Ferri R, Bella R, Ferini-Strambi L. The impact of drugs for multiple sclerosis on sleep. Mult Scler. 2017;23(1):5-13. doi:10.1177/ 1352458516664034

17. Tabrizi FM, Radfar M. Fatigue, sleep quality, and disability in relation to quality of life in multiple sclerosis. Int J MS Care. 2015;17 (6):268-274. doi:10.7224/1537-2073.2014-046

18. Nociti V, Losavio FA, Gnoni V, et al. Sleep and fatigue in multiple sclerosis: a questionnaire-based, cross-sectional, cohort study. J Neurol Sci. 2017;372:387-392. doi:10.1016/j.jns.2016.10.040 
19. Veauthier C, Radbruch H, Gaede G, et al. Fatigue in multiple sclerosis is closely related to sleep disorders: a polysomnographic cross-sectional study. Mult Scler. 2011;17(5):613-622. doi:10.1177/ 1352458510393772

20. Veauthier C, Gaede G, Radbruch H, Gottschalk S, Wernecke KD, Paul F. Treatment of sleep disorders may improve fatigue in multiple sclerosis. Clin Neurol Neurosurg. 2013;115(9):1826-1830. doi:10. 1016/j.clineuro.2013.05.018

21. Papuc E, Stelmasiak Z. Factors predicting quality of life in a group of Polish subjects with multiple sclerosis: accounting for functional state, socio-demographic and clinical factors. Clin Neurol Neurosurg. 2012;114(4):341-346. doi:10.1016/j.clineuro.2011.11.012

22. Sarraf P, Azizi S, Moghaddasi AN, Sahraian MA, Tafakhori A, Ghajarzadeh M. Relationship between sleep quality and quality of life in patients with multiple sclerosis. Int $J$ Prev Med. 2014;5 (12):1582-1586.

23. Leland NE, Marcione N, Schepens Niemiec SL, Kelkar K, Fogelberg D. What is occupational therapy's role in addressing sleep problems among older adults? OTJR: occup, participation health. 2014;34(3):141-149.

24. Perry M, Swain S, Kemmis-Betty S, Cooper P. Guideline development group of the national institute for $\mathrm{H}$, care E. multiple sclerosis: summary of NICE guidance. BMJ. 2014;349(oct08 10):g5701. doi:10.1136/bmj.g5701

25. Maitra K, Hall C, Kalish T, et al. Five-year retrospective study of inpatient occupational therapy outcomes for patients with multiple sclerosis. $\mathrm{Am}$ J Occup Ther. 2010;64(5):689-694. doi:10.5014/ajot.2010.090204

26. $\mathrm{Yu} \mathrm{CH}$, Mathiowetz V. Systematic review of occupational therapy-related interventions for people with multiple sclerosis: part 2. Impairment. Am J Occup Ther. 2014;68(1):33-38. doi:10.5014/ ajot.2014.008680

27. $\mathrm{Yu} \mathrm{CH}$, Mathiowetz V. Systematic review of occupational therapy-related interventions for people with multiple sclerosis: part 1. Activity and participation. Am J Occup Ther. 2014;68(1):27-32. doi:10.5014/ajot.2014.008672

28. American Occupational Therapy Association. Occupational therapy practice framework: domain and process (3rd ed.). Am J Occup Ther. 2014;68(Suppl. 1):S1-S48. doi:10.5014/ajot.2014.682006

29. Ansari NN, Naghdi S, Hasson S, Valizadeh L, Jalaie S. Validation of a Mini-Mental State Examination (MMSE) for the Persian population: a pilot study. Appl Neuropsychol. 2010;17(3):190-195. doi:10.1080/09084282.2010.499773

30. Farrahi Moghaddam J, Nakhaee N, Sheibani V, Garrusi B, Amirkafi A. Reliability and validity of the Persian version of the Pittsburgh Sleep Quality Index (PSQI-P). Sleep Breath. 2012;16 (1):79-82. doi:10.1007/s11325-010-0478-5
31. Heidari M, Akbarfahimi M, Salehi M, Nabavi SM. Validity and reliability of the Persian-version of fatigue impact scale in multiple sclerosis patients in Iran. koomesh. 2014;15(3):295-301.

32. Ho ECM, Siu AMH. Occupational therapy practice in sleep management: a review of conceptual models and research evidence. Occup Ther Int. 2018;2018:8637498. doi:10.1155/2018/8637498

33. Buysse DJ, Reynolds CF, Monk TH, Berman SR, Kupfer DJ. The Pittsburgh Sleep Quality Index: a new instrument for psychiatric practice and research. Psychiatry Res. 1989;28(2):193-213. doi:10. 1016/0165-1781(89)90047-4

34. Azimian M, Farahani AS, Dadkhah A, Fallahpour M, Karimlu M. Fatigue severity scale: the psychometric properties of the persian-version in patients with multiple sclerosis. Res J Biol Sci. 2009;4:974-977.

35. Ware JE, Sherbourne CD. The MOS 36-item short-form health survey (SF-36). I. Conceptual framework and item selection. Med Care. 1992;30(6):473-483. doi:10.1097/00005650-199206000-00002

36. Montazeri A, Goshtasebi A, Vahdaninia M, Gandek B. The Short Form Health Survey (SF-36): translation and validation study of the Iranian version. Qual Life Res. 2005;14(3):875-882. doi:10.1007/ s11136-004-1014-5

37. Folstein MF, Folstein SE, McHugh PR. "Mini-mental state". A practical method for grading the cognitive state of patients for the clinician. J Psychiatr Res. 1975;12(3):189-198. doi:10.1016/ 0022-3956(75)90026-6

38. Cohen J. Statistical Power Analysis for the Behavioral Sciences. 2nd ed. Routledge; 1988.

39. Dayapoglu N, Tan M. Evaluation of the effect of progressive relaxation exercises on fatigue and sleep quality in patients with multiple sclerosis. J Altern Complement Med. 2012;18(10):983-987. doi:10. 1089/acm.2011.0390

40. Attarian H, Applebee G, Applebee A, et al. Effect of eszopiclone on sleep disturbances and daytime fatigue in multiple sclerosis patients. Int J MS Care. 2011;13(2):84-90. doi:10.7224/1537-2073-13.2.84

41. Veauthier C, Gaede G, Radbruch H, Wernecke KD, Paul F. Sleep disorders reduce health-related quality of life in multiple sclerosis (Nottingham health profile data in patients with multiple sclerosis). Int J Mol Sci. 2015;16(7):16514-16528. doi:10.3390/ijms160716514

42. Wulff K, Gatti S, Wettstein JG, Foster RG. Sleep and circadian rhythm disruption in psychiatric and neurodegenerative disease. Nat Rev Neurosci. 2010;11(8):589-599. doi:10.1038/nrn2868
Neuropsychiatric Disease and Treatment

\section{Publish your work in this journal}

Neuropsychiatric Disease and Treatment is an international, peerreviewed journal of clinical therapeutics and pharmacology focusing on concise rapid reporting of clinical or pre-clinical studies on a range of neuropsychiatric and neurological disorders. This journal is indexed on PubMed Central, the 'PsycINFO' database and CAS, and is the official journal of The International Neuropsychiatric Association (INA). The manuscript management system is completely online and includes a very quick and fair peer-review system, which is all easy to use. Visit http://www.dovepress.com/testimonials.php to read real quotes from published authors. 